(C) 2014 IEEE. Personal use of this material is permitted. Permission from IEEE must be obtained for all other uses, in any current or future media, including reprinting/republishing this material for advertising or promotional purposes, creating new collective works, for resale or redistribution to servers or lists, or reuse of any copyrighted component of this 


\title{
An Automated Mechanism to Characterize Wheelchair User Performance
}

\author{
Bojan Andonovski ${ }^{1}$, Jaime Valls Miro ${ }^{1}$, James Poon ${ }^{1}$ and Ross Black ${ }^{2}$
}

\begin{abstract}
This paper proposes a mechanism to derive quantitative descriptions of wheelchair usage as a tool to aid Occupational Therapist with their performance assessment of mobility platform users. This is accomplished by analysing data computed from a standalone sensor package fitted on an wheelchair platform. This work builds upon previous propositions where parameters that could assist in the assessment were recommended to the authors by a qualified occupational therapist (OT). In the current scheme however the task-specific parameters that may provide the most relevant user information for the assessment are automatically revealed through a machine learning approach. Data mining techniques are used to reveal the most informative parameters, and results from three typical classifiers are presented based on learnings from manual labelling of the training data. Trials conducted by healthy volunteers gave classifications with an $81 \%$ success rate using a Random Forest classifier, a promising outcome that sets the scene for a potential clinical trial with a larger user pool.
\end{abstract}

\section{INTRODUCTION}

Powered mobility devices (PMDs) such as electric wheelchairs and scooters are popular ambulation devices used by the aged and disabled population. Adults aged over 50 years are the most prevalent wheelchairs users [1], and it is estimated that PMD use is 3.5 times more frequent after the age of 65 [2]. There are over 4.3 million users of powered wheelchairs in the US alone [2], and it has been reported that $10 \%$ of powered wheelchair users experience serious difficulties with the standard operation of their wheelchair [1]. Furthermore, there are many other individuals who require mobility assistance yet also have other conditions such as visual or cognitive impairments, that hamper their ability to safely operate a powered wheelchair. These factors coupled with new technologies providing a broad range of wheelchair options complicate the mobility aid prescription process [3], resulting in an increasing need for an outcome measurement to clinically quantify the necessity of mobility aids from one patient to another.

Mobility performance measures have been mostly proposed in the literature as a mean to provide some form of shared control of the platform. The work presented as part of the "CanWheel" project [4] shows the outcomes of Intelligent Wheelchair System (IWS) developed to help older adults with cognitive impairments drive a powered

\footnotetext{
${ }^{1}$ Bojan Andonovski, Jaime Valls Miro and James Poon are with the Faculty of Engineering and IT, University of Technology Sydney (UTS), Sydney NSW 2007, Australia bojan.andonovski@uts.edu.au, jaime.vallsmiro@uts.edu.au,

${ }^{2}$ Ross Black is with the Occupational Therapy Department, Prince of Wales Hospital, Sydney NSW 2031, Australia ross.blackesesiahs.health.nsw.gov.au
}

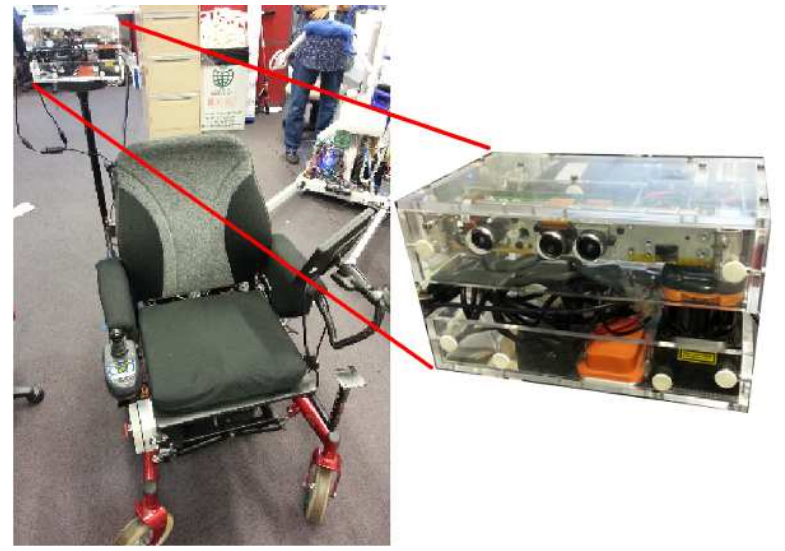

Fig. 1: Power wheelchair platform with close-up of situational monitoring sensor package developed for this work.

wheelchair safely and effectively. Past work on the profiling of wheelchair users [5] has also been done with focus on tailoring aspects of collaborative control [6], and scenariobased profiling has proven suitable for potentially long-term co-autonomy [7]. Similar mobility performance measures have also been trialled as a means for comparison with the widely used Wheelchair Skills Test (WST) with promising results [8]. However, this may not be practical for professional classification in a clinical setting where many patients may be testing for prescription, given possible limitations on both time and the quantity of available test equipment. A series of brief, easily repeated tasks draws a closer parallel to currently practiced clinical methods, as they possess a greater ease of use for therapists due to their inherent simplicity.

In disability healthcare there exist multiple means for determining performance of mobility aid users such as the WST [9] mentioned above, and the Power-Mobility Indoor Driving Assessment (PIDA) [10]. The outcomes of these tests normally depend on the judgment of a qualified occupational therapist (OT) with recommendations [11] on the most suitable type of mobility aid, if any. Since there are many tests with different assessment standards, inconsistent scoring often results even under similar test scenarios [12]. Despite desirable increases to assessment consistency and efficiency there is yet to be a means for automated assessment based on criteria from standard wheelchair assessment tasks, in a format that is also convenient to therapist staff overseeing patient rehabilitation. Work towards this alternative such as the use of algorithm-derived questionnaires when objective testing was infeasible has been conducted [13], but self- 


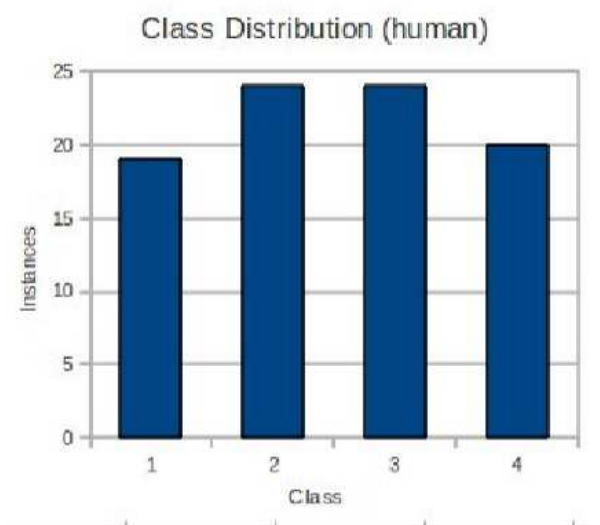

Fig. 2: Recorded data distribution for $10 \mathrm{~m}$ runs.

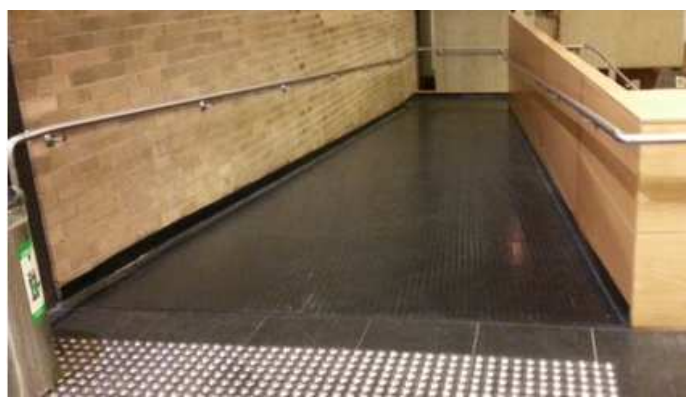

Fig. 3: Test ramp (6º gradient).

assessments are likely to carry a tendency towards users overestimating their own navigational capacities [14].

There is also much work in the literature covering the use of machine learning algorithms in a vast range of classification applications from cancer diagnosis criteriato the analysis of soccer videos.Classification analysis through data mining techniques is also becoming widely adopted for healthcare applications given the large bodies of data to decide what information is most relevant to improve quality of patient care. Support Vector Machine (SVM) and K-Nearest Neighbors (KNN) have been compared to guide rehabilitation planning for home care clients [15] for instance. However, little has been done to study whether a machine learning engine could provide support to therapists in determining what the most appropriate PMD for a client could be, a judgement in itself compounded by the lack of agreement in the tests to be carried out to decipher such outcome. The incipient results in this paper point to the fact that machine learning approaches have got the potential to serve as a clinical assessment tool to replicate the judgement of a therapist in efficient and consistent mobility aid performance classification. To that end, a methodology is proposed to find correlations between therapist scores and quantitative measures that can be evaluated with a standalone sensor package. Whereas our earlier works [16], [17] were based on environmental attributes such as alignment with a bed and other parameters recommended by a therapist, the work hereby presented proposes the use of parameters

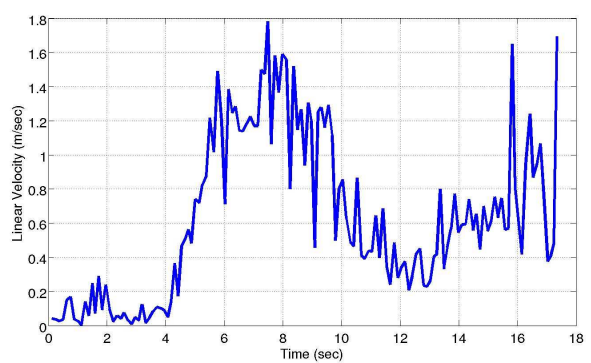

Fig. 4: Sample linear velocity profile for $10 \mathrm{~m}$ task (class 1 ).

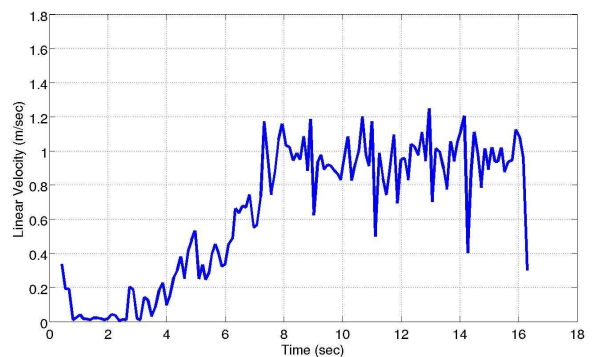

Fig. 5: Sample linear velocity profile for $10 \mathrm{~m}$ task (class 4).

data-mined from test runs for an assessment of general user proficiency. This is done in order to be unrestricted to any particulars concerning the required assessment outcomes of the respective OT, allowing classification to focus solely on navigational ability. Selection of parameters based on platform movement is done through machine learning, as parameters possibly less intuitive to interpret may possibly provide greater insights for classification. After this preprocessing step, a number of discriminative classifiers are studied to analyse the skill of a PMD user.

The remainder of the paper is as follows: a description of the experimental setup is given in Section II, with an outline of the process undertaken for identification of useful measurements described in Section III. Classification experiments are covered in Section IV with results, discussion and concluding remarks in Sections V, VI and VII respectively.

\section{Experiment Setup and Data Collection}

This paper is primarily concerned on parameters gathered from short driving activities as a way to assess the validity of the proposed scheme. As such, three representative tasks were selected out of PIDA's available 35, namely " $180^{\circ}$ turn", "driving on an indoors incline" and "10m forward driving" tasks. An instrumented wheelchair platform (Fig 1) equipped with drive motors and wheel encoders was fitted with a modular sensor package housing an RGB-D camera (MS Kinect), a Hokuyo laser range finder and an Xsens inertial measurement unit (IMU). The sensors were all connected with a wheelchair's on-board PC controller. More information detailing the wheelchair and sensor package can be found in [16].

Multiple runs were then recorded from five able users while they performed the tasks under controlled environmental conditions. Details are collected in Table I. The use of 
TABLE I: Task Datasets

\begin{tabular}{c|c|c}
\hline Experiment & Number of Datasets & Number of Test Users \\
\hline $10 \mathrm{~m}$ drive & 78 & 4 \\
$180^{\circ}$ turn & 78 & 5 \\
Ramp drive & 61 & 4 \\
\hline
\end{tabular}

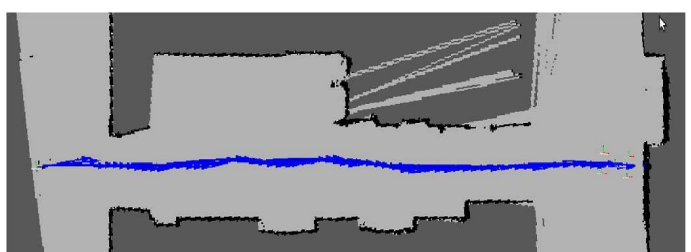

Fig. 6: Sample 10m run.

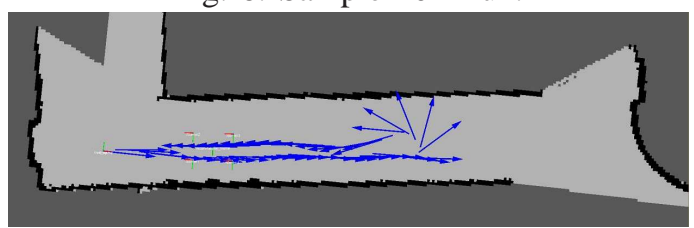

Fig. 7: Sample $180^{\circ}$ turn.

nave able bodied users and limited data sets to demonstrate proof-of-concept are considered a necessary first step before seeking to apply the methods to those with a disability. Able bodies users will, like those with a disability, exhibit a range of skill levels that, while neccesarily different from those with a dissability, can still potentially be identified using the proposed machine learning techniques. Nonetheless, to align the skill set with those of the intended audience, users were asked to simulate varying degrees of erraticness in their driving, and were labelled accordingly. This is of course rather subjective, but in essence so is the task at hand, as what makes a "proficient, or "bad" driver of a power mobility device is hard to discern in itself. But is is precisely the capacity of the proposed techniques to capture and model variability in the data trends, particularly given the associated inherent noise in the measurements, that this work is trying to capture. As per the PIDA scale, the four classes range from poor (1) to proficient (4). Figure 2 shows the spread of the datasets collected, with classes distributed roughly equally and a slightly lesser number of runs deemed to be very poor or very good. Two samples of one of the extracted parameters from the $10 \mathrm{~m}$ run task, in this case the linear velocity profile, are shown in Figures 4 and 5 for class 1 and 4 respectively (further details about the parameters analysed will be given in Section III). It can be observed how the class 4 profile is relatively smoother despite some peaks towards the end of the run, whereas the class 1 profile appears more erratic throughout.

All the development was done in the Robot Operating System (ROS) software environment (www.ros.org). Laser scanner, odometry and IMU data was fused to map and provide wheelchair localization during the runs via the Hector mapping package [18]. Additional ROS support was developed for each of the tasks and time-stamped parameters (e.g. linear and angular velocity, proximity to obstacles,

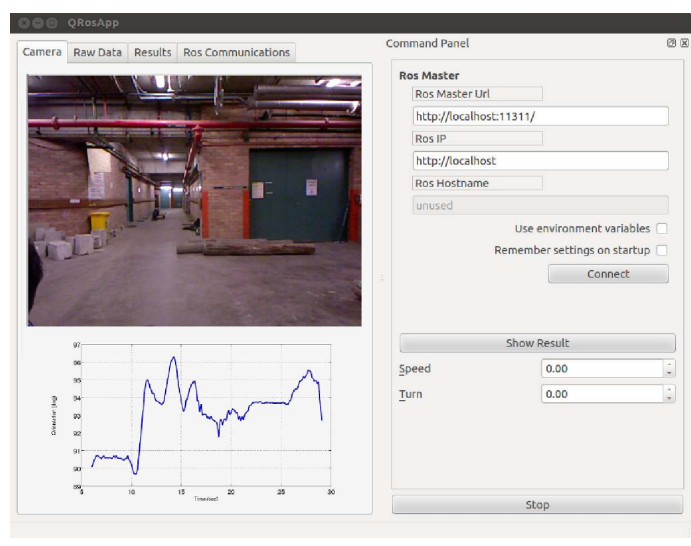

Fig. 8: Assessment GUI.

etc) were recorded during the user trials. Figures 6 and 7 depict a sample wheelchair trajectory from a run recorded for the $10 \mathrm{~m}$ and $180 \mathrm{deg}$ task repectively. The arrows indicate platform position and orientation, with points identified to be solid objects by the laser scanner (such as walls) shown in black. Light-grey areas represent known empty space, and the remaining dark-grey space are the unknown regions beyond the environment sensed by the scanner. Figure 3 shows the ramp used for ramp task simply for illustration. Figure 8 depicts the simple GUI developed with the future assessing staff in mind to be able to conveniently use the system in an intuitive, repetitive and consistent manner with little need for familiarization with the underlying robotics hardware or software. This was done with the intention to allow for more tests to be conducted efficiently in a clinical setting. The interface is Qt-based and uses a MATLAB pipeline behindthe-scenes for classification based on incoming data bridged from the ROS sensor drivers.

\section{PARAMETER Selection}

Various features were extracted from the sensor data and used as an input to the classifier to perform the assessments. Parameter selection was carried out using the Attribute Selection feature within the WEKA toolkit [19] to find the most informative attributes first. The evaluator approach computes the intrinsic value of a subset of attributes by considering the individual predictive ability of each feature, along with the degree of redundancy between them. Among the considerable number of quantitative measurements which could be derived from the sensor package data, a number of key metrics were selected as most representative for each of the tasks. Hence, for the $10 \mathrm{~m}$ driving task the following parameters were used:

1) Average linear velocity

2) Standard deviation of linear velocity $(*)$

3) Average angular velocity

4) Standard deviation of angular velocity (*)

5) Time to accomplish the task

Seven metrics were selected for the $180^{\circ}$ turning task:

1) Average linear velocity

2) Standard deviation of linear velocity 
TABLE II: 10m Task Parameter Statistics

\begin{tabular}{c|ccccc}
\cline { 2 - 6 } & \multicolumn{5}{c}{ Parameter } \\
& 1 & 2 & 3 & 4 & 5 \\
\hline Min & 0.36 & 0.17 & 0.02 & 0.01 & 5.77 \\
Max & 1.72 & 4.16 & 0.72 & 0.33 & 27.89 \\
Mean & 0.72 & 1.02 & 0.27 & 0.12 & 15.68 \\
Std Dev. & 0.30 & 0.65 & 0.19 & 0.08 & 4.85 \\
\hline
\end{tabular}

TABLE III: $180^{\circ}$ Turn Task Parameter Statistics

\begin{tabular}{c|ccccccc}
\cline { 2 - 8 } & \multicolumn{7}{c}{ Parameter } \\
& 1 & 2 & 3 & 4 & 5 & 6 & 7 \\
\hline Min & 0.07 & 0.15 & 0.03 & 0.07 & 7.36 & 5.05 & 0.04 \\
Max & 0.82 & 0.72 & 0.63 & 0.32 & 45.13 & 39.0 & 0.35 \\
Mean & 0.38 & 0.37 & 0.24 & 0.14 & 19.83 & 16.02 & 0.11 \\
Std Dev. & 0.17 & 0.13 & 0.17 & 0.07 & 9.73 & 8.78 & 0.09
\end{tabular}

TABLE IV: Ramp Task Parameter Statistics

\begin{tabular}{c|ccccccc}
\cline { 2 - 8 } & \multicolumn{7}{c}{ Parameter } \\
& 1 & 2 & 3 & 4 & 5 & 6 & 7 \\
\hline Min & 0.13 & 0.11 & 0.04 & 0.02 & 3.19 & 0.03 & 3.66 \\
Max & 1.74 & 2.63 & 0.71 & 0.68 & 26.89 & 0.26 & 7.69 \\
Mean & 0.82 & 0.45 & 0.25 & 0.19 & 10.66 & 0.08 & 4.69 \\
Std Dev. & 0.36 & 0.38 & 0.20 & 0.14 & 4.46 & 0.04 & 1.82 \\
\hline
\end{tabular}

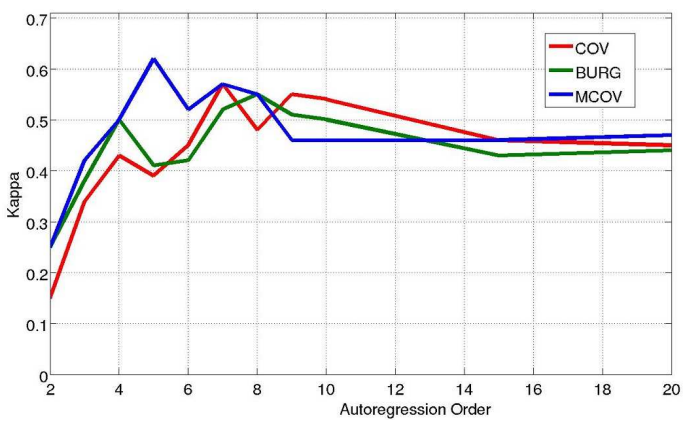

Fig. 9: Kappa values from parametric transform

3) Average angular velocity

4) Standard deviation of angular velocity (*)

5) Time to accomplish the task

6) Idle time

7) Minimum distance to obstacle

And a further seven metrics were selected for the ramp driving task:

1) Average linear velocity

2) Standard deviation of linear velocity

3) Average angular velocity

4) Standard deviation of angular velocity (*)

5) Time to accomplish the task

6) Minimum distance to obstacle

7) Gradient of incline

Tables II-IV provide a breakdown of each tasks' parameters. $\left.{ }^{*}\right)$ denotes the most informative parameters identified for each of the tasks. The standard deviation of angular velocity was the most informative parameter identified for all three tasks, being the sole parameter for the ramp and $180^{\circ}$ tasks. The $10 \mathrm{~m}$ driving task also had the standard deviation of linear velocity as an additional distinctive metric.

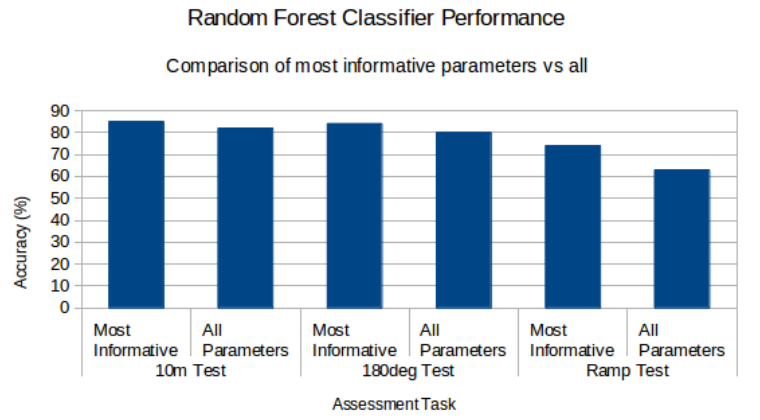

Fig. 10: Summary of results for best classifier on the 3 tasks.

\section{Classification Methodology}

Two methods were attempted to classify data: the first relied on time-dependent features such as velocities and derivations thereof such as mean and standard deviation. The second approach was to change the domain of the data via parametric transformations for feature matrices of burg, covariance and modified covariance [20] between autoregression orders 2 to 20 (Fig 9). The upper limit of the autoregression range was manually determined due to the convergence of kappa values following order 15, which would provide less useful information. Parametric transforms use orders of autoregression, a magnitude of derived weighted sum of previous to current values. In that way the data representation (domain) is changed into specific time windows, to see if they can provide greater information compared to a dataset in its entirety. As the data is interpreted through a different representation, a new set of parameters is also obtained which may be more beneficial to classification compared to their time-domain counterparts. Burg, covariance and modified covariance methods are all autoregression processes with different approaches for estimating data strength.

All available classifiers within WEKA were trained with datasets randomly selected from all 4 classes and were compared using both time-dependent features and feature matrices from parametric transformation for two approaches to analysis. Each classifier was tested using cross-validation in WEKA utilizing six 'folds': all available data for a task is divided into six sets, and a classifier is trained from five sets and applied to the remaining one. This is repeated for each set, resulting in six classifiers whose results are aggregated for the overall performance of the classifier encompassing the task's available data pool.

\section{RESUlts}

Random Forest (RF) and Support Vector Machine (SVM) yielded the best results using time-dependent data, and their confusion matrices are shown in Tables V and VI with the best classifier's results graphically represented in Figure 10. Random Forest classification uses a number of generated decision maps or 'trees', that branch from a random set of features at each decision point. This classifier attempts to minimize both its own bias and the spread of data, making it well-suited for classification with a limited number of 
TABLE V: Time-domain Random Forest

\begin{tabular}{c|cccc|cccc|cccc}
\cline { 2 - 12 } & \multicolumn{4}{c}{$10 \mathrm{~m}$ task } & \multicolumn{4}{c|}{$180^{\circ}$ turn task } & \multicolumn{4}{c}{ Ramp task } \\
& 1 & 2 & 3 & 4 & 1 & 2 & 3 & 4 & 1 & 2 & 3 & 4 \\
\hline 1 & 16 & 1 & 0 & 0 & 17 & 1 & 0 & 0 & 12 & 1 & 1 & 0 \\
2 & 1 & 21 & 0 & 0 & 1 & 19 & 1 & 1 & 2 & 10 & 1 & 1 \\
3 & 0 & 1 & 19 & 1 & 0 & 1 & 19 & 1 & 2 & 1 & 18 & 2 \\
4 & 1 & 2 & 1 & 14 & 1 & 0 & 2 & 14 & 0 & 0 & 0 & 10 \\
\hline
\end{tabular}

TABLE VI: Time-domain Support Vector Machine

\begin{tabular}{c|cccc|cccc|cccc}
\cline { 2 - 12 } & \multicolumn{4}{c}{$10 \mathrm{~m}$ task } & \multicolumn{4}{c|}{$180^{\circ}$ turn task } & \multicolumn{4}{c}{ Ramp task } \\
& 1 & 2 & 3 & 4 & 1 & 2 & 3 & 4 & 1 & 2 & 3 & 4 \\
\hline 1 & 15 & 2 & 0 & 0 & 16 & 1 & 1 & 0 & 11 & 2 & 1 & 0 \\
2 & 2 & 18 & 1 & 1 & 2 & 15 & 2 & 3 & 2 & 9 & 1 & 2 \\
3 & 0 & 3 & 16 & 2 & 0 & 1 & 19 & 1 & 1 & 2 & 18 & 2 \\
4 & 1 & 1 & 3 & 13 & 0 & 2 & 2 & 13 & 0 & 0 & 0 & 10 \\
\hline
\end{tabular}

TABLE VII: Parametric Random Forest

\begin{tabular}{c|cccc|cccc|cccc}
\cline { 2 - 14 } & \multicolumn{4}{c}{$10 \mathrm{~m}$ task } & \multicolumn{4}{c|}{$180^{\circ}$ turn task } & \multicolumn{4}{c}{ Ramp task } \\
& 1 & 2 & 3 & 4 & 1 & 2 & 3 & 4 & 1 & 2 & 3 & 4 \\
\hline 1 & 14 & 1 & 1 & 0 & 15 & 1 & 1 & 1 & 10 & 2 & 2 & 0 \\
2 & 1 & 21 & 0 & 0 & 1 & 17 & 2 & 2 & 2 & 10 & 1 & 1 \\
3 & 1 & 4 & 14 & 2 & 1 & 2 & 17 & 1 & 2 & 1 & 18 & 2 \\
4 & 2 & 2 & 3 & 12 & 1 & 1 & 3 & 12 & 0 & 0 & 0 & 10 \\
\hline
\end{tabular}

features and datasets, as is the case in our experiments. SVM is a widely-used classifier that requires manual classification of some data before automatic classification can proceed. Training results in a set of data that is 'learned', which can then be used as the basis of a statistical model for assigning the remaining data into classes. Table VII shows resultant confusion matrices from the best of the parametric transformation approaches: a Random Forest classifier on Modified Covariance with autoregression order 5.

Table VIII displays percentage accuracies for each method using the most evaluated parameters in comparison with all available parameters, for the approaches from the confusion matrices. Table IX shows values for Cohen's kappa [21], a measurement of inter-rater agreement for qualitative attributes. This value provides an indication of how far a classifier varies from the diagonal in a confusion matrix between 0 (poor) and 1 (perfect). It can be seen that overall the Random Forest on time-domain data was the most successful classification method for the three assessment tasks, by achieving an averaged $81 \%$ classification rate compared to $75 \%$ and $72 \%$ for the SVM and parametric Random Forest methods respectively. The parametric transform approach did not perform as well as the time-domain classification due to the narrow distribution of data based on time-domain parameters. Similarly the SVM's performance was inferior to that of the time-domain Random Forest, owing to the limited number of features for learning. It is also shown in Table VIII that resultant accuracies for only selecting the most informative criteria were unanimously higher than when all parameters were used. This outcome is reinforced with an averaged Cohen's kappa of 0.67 from the Random Forest method on time-domain data, indicating a strong relevance.

Resulting confusion matrices of a comparison with our earlier work [17] are collected in Table X. In this case the "Speed Selection" of the PIDA test was the task of
TABLE VIII: Accuracy (\%)

\begin{tabular}{c|c|ccc}
\cline { 3 - 5 } \multicolumn{2}{c}{} & RF (time) & SVM & RF (parametric) \\
\hline \multirow{2}{*}{$10 \mathrm{~m}$} & Most Inf. & 85 & 83 & 72 \\
& All Param. & 82 & 82 & 68 \\
\hline \multirow{2}{*}{$180^{\circ}$ turn } & Most Inf. & 84 & 73 & 73 \\
& All Param. & 80 & 70 & 71 \\
\hline \multirow{2}{*}{ Ramp } & Most Inf. & 74 & 70 & 70 \\
& All Param. & 63 & 61 & 60 \\
\hline
\end{tabular}

TABLE IX: Cohen's Kappa

\begin{tabular}{c|c|ccc}
\cline { 3 - 5 } \multicolumn{2}{c}{} & RF (time) & SVM & RF (parametric) \\
\hline \multirow{2}{*}{$10 \mathrm{~m}$} & Most Inf. & 0.71 & 0.62 & 0.62 \\
& All Param. & 0.67 & 0.59 & 0.6 \\
\hline \multirow{2}{*}{$180^{\circ}$ turn } & Most Inf. & 0.69 & 0.6 & 0.61 \\
& All Param. & 0.68 & 0.57 & 0.6 \\
\hline \multirow{2}{*}{ Ramp } & Most Inf. & 0.6 & 0.59 & 0.58 \\
& All Param. & 0.51 & 0.5 & 0.49 \\
\hline
\end{tabular}

interest. For this scenario, the ability of the user to select the appropriate speed with respect to the environment as observed throught the PIDA test is the OT's primary subjective criteria in their assessment. The task was restricted in that work to navigating through a large room, opening a door and parking alongside a bed. To establish the correlation with the methodology hereby proposed, the three tasks were combined into 61 longer, amalgamated runs and labelled based on an overall score of speed selection for each of the runs. Table XI depicts obtained accuracy and Cohen's kappa of the speed selection classifiers for amalgamated runs. There are only slightly differences between speed selection classifiers values on accuracies and kappa. The use of most informative parameters to classify the whole performance of the users run outperformed the accuracy when using only speed selection for classification. This, point out that the identified parameters may indeed be helpful to OT staff.

\section{DISCUSSION}

Despite the shortcoming of a limited set of experiments conducted on able bodies, the results obtained appear promising to address a clear need. Selecting the most appropriate PMD for a client is a time intensive and financially costly process. If therapists can have access to a critical or minimum data set a client will still obtain the most appropriate PMD, spend less time in therapy and give the therapist an opportunity to target those parameters which are critical for a persons success in operating the PMD. Novice therapists using machine learning systems could potentially function at the same level as expert therapists using conventional techniques. Future work will focus on comparing use of machine learning systems and current assessment techniques. In addition, therapists would have an opportunity to examine users performance "in silico" to determine their skill level and if their skill is improving in response to the therapists input. Machine learning systems could assist therapists to extend the evidence-base of their practice, reduce the amount of time taken to learn and perform mobility aid assessments, improve the quality of assessments and reflect on the quality of their assessment techniques and tools. The professional and financial benefits would be considerable. 
TABLE X: Speed selection for combined tasks

\begin{tabular}{c|cccc|cccc|cccc}
\cline { 2 - 18 } & \multicolumn{4}{c|}{ RF (time) } & \multicolumn{4}{c|}{ SVM } & \multicolumn{4}{c}{ RF (parametric) } \\
& 1 & 2 & 3 & 4 & 1 & 2 & 3 & 4 & 1 & 2 & 3 & 4 \\
\hline 1 & 1 & 4 & 0 & 0 & 2 & 3 & 0 & 0 & 2 & 2 & 1 & 0 \\
2 & 1 & 9 & 5 & 2 & 1 & 12 & 2 & 2 & 2 & 11 & 2 & 2 \\
3 & 0 & 4 & 14 & 4 & 0 & 3 & 14 & 5 & 1 & 1 & 15 & 5 \\
4 & 0 & 0 & 5 & 12 & 0 & 0 & 7 & 10 & 1 & 1 & 9 & 6 \\
\hline
\end{tabular}

TABLE XI: Accuracy and Cohen's Kappa for speed selection classification

\begin{tabular}{c|ccc}
\cline { 2 - 4 } & RF (time) & SVM & RF (parametric) \\
\hline Accuracy (\%) & 59 & 62 & 56 \\
Cohen's Kappa & 0.41 & 0.46 & 0.4 \\
\hline
\end{tabular}

In this work the initial subset of parameters have been chosen to be more aligned with those currently/usually/readily/typically assessed by therapists in their tests such as velocity and time taken to accomplish a task. It would be however very useful to assess others, e.g. trajectory profile, acceleration, or the derivative of acceleration (jerk) which is generally understood to determine comfort in driving. Interestingly, by studying for instance the relevance of a parameters such as jerk, one would be able to gain an insight into whether PMD users drive their chairs in such a way as to reduce jerk. If so, it underlines the use of machine learning systems and use of a stand-alone sensor package as no therapist is going to be able to determine chair jerk during a subjective observation and pencil and paper recording of results. This study has been left for future work.

\section{CONCLUSION}

The paper describes a method of efficiently interpreting data derived from a stand-alone sensor package mounted on an electric wheelchair (or PMD). The technique is of potential use to Occupational Therapists, who perform assessments of those who use electric wheelchairs (or PMDs), as it would allow them to critically analyse the objective data obtained from the sensor package during an assessment. Data mining techniques have been investigated to establish correlations across three standard assessment tasks with experimental data, achieving an $81 \%$ accuracy with a Random Forest classifier. The experiments demonstrate consistent agreement between the objective data acquired from the sensing array and the subjective assessments of a human in analysing the driving skills of a wheelchair user. These outcomes present occupational therapists with additional utilities to help discern whether patients are ready to be safely deployed with mobility aids for their daily activities. Despite the shortcomings of the experiments we believe the results remain relevant in the context of this research, and we hope to continue with a clinical trial involving a broader user base of disabled individuals over a greater variety of assessment tasks along with the insight of qualified OT staff.

\section{ACKNOWLEDGMENT}

This work is based on a collaboration between the Centre for Autonomous Systems at the University of Technology Sydney, and the Prince of Wales Hospital in Sydney.

\section{REFERENCES}

[1] P. Clarke and A. Colantonio, "Wheelchair use among community dwelling older adults: prevalence and risk factors in a national sample," Canadian Journal on Aging, vol. 24, pp. 191-198, 2005.

[2] H. S. Kaye, T. Kang, and M. P. LaPlante, Mobility Device Use in the United States. Disability Statistics Report 14. Disability Statistics Center, University of California, San Francisco, 2000.

[3] W. B. Mortenson, W. C. Miller, and C. Auger, "Issues for the selection of wheelchair-specific activity and participation outcome measures: a review." Archives of Physical Med. and Rehab., vol. 89, no. 6, pp. 1177-1186, 2008.

[4] P. Viswanathan, J. J. Little, A. K. Mackworth, and A. Mihailidis, "Navigation and obstacle avoidance help (noah) for older adults with cognitive impairment: A pilot study," in ACM SIGACCESS Conf. on Computers and Accessibility, 2011, pp. 43-50.

[5] C. Urdiales, E. Perez, G. Peinado, M. Fdez-Carmona, J. Peula, R. Annicchiarico, F. Sandoval, and C. Caltagirone, "On the construction of a skill-based wheelchair navigation profile," IEEE Trans. on Neural Systems and Rehab. Eng., vol. 21, no. 6, pp. 917-927, Nov 2013.

[6] C. Urdiales, M. Fernandez-Carmona, J. M. Peula, U. Cortes, R. Annichiaricco, C. Caltagirone, and F. Sandoval, "Wheelchair collaborative control for disabled users navigating indoors," Artificial Intelligence in Med., vol. 53, no. 3, pp. 177-191, 2011.

[7] G. Peinado, C. Urdiales, J. M. Peula, M. Fdez-Carmona, R. Annicchiarico, F. Sandoval, and C. Caltagirone, "Navigation skills based profiling for collaborative wheelchair control," in IEEE Intl. Conf. on Robotics and Automation, May 2011, pp. 2229-2234.

[8] D. Pradon, N. Pinsault, Z.Raphal, and R.Franois, "Could mobilty performance measures be used to evaluate wheelchair skills?" Journal of Rehab. Med., vol. 44, no. 3, pp. 276-279, 2012.

[9] R. L. Kirby, J. Swuste, D. J. Dupuis, D. A. MacLeod, and R. Monroe, "The wheelchair skills test: A pilot study of a new outcome measure," Archives of Physical Med. and Rehab., vol. 83, no. 1, pp. 10-18, 2002.

[10] D. Dawson, R. Chan, and E. Kaiserman, "Development of the powermobility indoor driving assessment for residents of long-term care facilities: A preliminary report," Canadian Journal of Occupational Therapy, vol. 61, no. 5, pp. 269-276, 1994.

[11] K. Huhn, P. Guarrera-Bowlby, and J. E. Deutsch, "The clinical decision-making process of prescribing power mobility for a child with cerebral palsy," Pediatric Physical Therapy, vol. 19, no. 3, pp. 254-260, 2007.

[12] O. Fliess-Douer, Y. C. Vanlandewijck, G. L. Manor, and L. H. V. D. Woude, "A systematic review of wheelchair skills tests for manual wheelchair users with a spinal cord injury: towards a standardized outcome measure," Clinical Rehab., vol. 24, no. 10, pp. 867-886, 2010.

[13] A. D. Mountain, R. L. Kirby, and C. Smith, "The wheelchair skills test, version 2.4: Validity of an algorithm-based questionnaire version," Archives of Physical Med. and Rehab., vol. 85, no. 3, pp. 416-423, 2004.

[14] P. W. Rushton, R. L. Kirby, and W. C. Miller, "Manual wheelchair skills: objective testing versus subjective questionnaire," Archives of Physical Med. and Rehab., vol. 93, no. 12, pp. 2313-2318, 2012.

[15] M. Zhu, Z. Zhang, J. P. Hirdes, and P. Stolee, "Using machine learning algorithms to guide rehabilitation planning for home care clients," BMC Medical Informatics and Decision Making, vol. 7, no. 41, pp. 1-13, 2007.

[16] J. V. Miro, R. Black, F. de Bruijn, and G. Dissanayake, "Semiautonomous competency assessment of powered mobility device users," in IEEE Intl. Conf. on Rehab. Robotics, 2011, pp. 1-6.

[17] J. V. Miro, R. Black, B. Andonovski, and G. Dissanayake, "Development of a novel evidence-based automated powered mobility device competency assessment," in IEEE Intl. Conf. on Rehab. Robotics, 2013, pp. 1-8.

[18] S. Kohlbrecher, J. Meyer, O. von Stryk, and U. Klingauf, "A flexible and scalable slam system with full $3 \mathrm{~d}$ motion estimation," in IEEE Intl. Symp. on Safety, Security, and Rescue Robotics, 2011, pp. 155-160.

[19] M. Hall, E. Frank, G. Holmes, B. Pfahringer, P. Reutemann, and I. H. Witten, "The weka data mining software: an update," SIGKDD Explorations, vol. 11, no. 1, pp. 10-18, 2009.

[20] A. Zaknich, Principles of Adaptive Filters and Self-learning Systems. Springer, 2000.

[21] J. Cohen, "A coefficient of agreement of nominal scales," Educational and Psychological Measurement, vol. 20, pp. 37-46, 1960. 